J. DIFFERENTIAL GEOMETRY

82 (2009) 363-382

\title{
STRONG UNIQUENESS OF THE RICCI FLOW
}

\author{
BING-LONG CHEN
}

\begin{abstract}
In this paper, we derive some local a priori estimates for the Ricci flow. This gives rise to some strong uniqueness theorems. As a corollary, let $g(t)$ be a smooth complete solution to the Ricci flow on $\mathbb{R}^{3}$, with the canonical Euclidean metric $E$ as initial data, then $g(t)$ is trivial, i.e. $g(t) \equiv E$.
\end{abstract}

\section{Introduction}

The Ricci flow $\frac{\partial}{\partial t} g_{i j}(x, t)=-2 R_{i j}(x, t)$, was introduced by Hamilton in [7]. The major application of this equation to lower dimensional topology has had a great impact in modern mathematics (see [7], [8], [9] [12], [13]). The power of these geometric applications grew out of the fundamental PDE theory of the equation. These two aspects had been intertwined all the time since the foundation of the Ricci flow.

In this paper, we go back to some fundamental PDE problems of this equation.

Let's look at one heuristic analogue, the standard heat equation $\left(\frac{\partial}{\partial t}-\triangle\right) u=0$ on $\mathbb{R}^{n}$. If $u$ grows slower than the function $e^{a|x|^{2}}$ for some $a>0$, then $u$ is unique for all such solutions with the same initial data. Moreover, if $\left.|u|\right|_{t=0} \leq C e^{a|x|^{2}}$, it is not hard to see the short time existence (of solutions of same type) from the heat kernel convolution. For the Ricci flow, the Ricci curvature behaves like twice derivative of logarithmic of the metric. So bounded curvature condition for the Ricci flow resembles growth $e^{a|x|^{2}}$ for the standard heat equation. Actually, the fundamental work [14] showed that on complete manifolds with bounded curvature, the Ricci flow always admits short time solutions of bounded curvature. X.P. Zhu and the author recently [3] proved that the uniqueness theorem holds for solutions in the class of bounded curvature. For an interesting application of this theorem to the theory of Ricci flow with surgery, we refer the readers to see [4] or relevant discussions in $[\mathbf{1}][\mathbf{1 0}][\mathbf{1 1}]$.

This work was partially supported by grants from Sun Yat-Sen University NCET050717, N0. 34000-3171404 \& 34000-1131040, 973 project 2006CB805905 and NSFC 10831008.

Received 06/01/2007. 
However, if one don't impose any growth conditions, the solutions to the heat equation $\left(\frac{\partial}{\partial t}-\triangle\right) u=0$ are no longer unique. For instance, when $n=1$, the famous Tychonoff's example

$$
u(x, t)=\sum_{k=0}^{\infty} \frac{x^{2 k}}{(2 k) !} \frac{d^{k}}{d t^{k}} e^{-\frac{1}{t^{2}}},
$$

is a smooth nontrivial solution to the heat equation with 0 initial data. The purpose of this paper is to investigate the analogous problem for the Ricci flow. Nevertheless, the Ricci flow, as the most natural intrinsic heat deformation of metrics, has quite complicated nonlinearity. We attempt to show that, in certain extent, the above phenomenon will not happen for geometrically reasonable solutions in dimension 3 .

Now, we formulate one of the main results of this paper as following

Theorem 1.1. Let $(M, g(x))$ be a complete noncompact three dimensional manifold with bounded and nonnegative sectional curvature $0 \leq$ $R m \leq K_{0}$, for some fixed constant $K_{0}$. Let $g_{1}(x, t), g_{2}(x, t), t \in[0, T]$, be two smooth complete solutions to the Ricci flow with initial data $g(x)$. Then we have $g_{1}(t) \equiv g_{2}(t)$, for $0 \leq t<\min \left\{T, \frac{1}{4 K_{0}}\right\}$.

A simple example is the Euclidean space $\mathbb{R}^{3}$ :

Corollary 1.2. Let $g(t), t \in[0, T]$, be a smooth complete solution to the Ricci flow on $\mathbb{R}^{3}$, starting with the canonical Euclidean metric E, then $g(t) \equiv E$.

The most important feature of these uniqueness theorems, is that we do not require any extra growth conditions on the solutions except the geodesic completeness.

Theorem 1.1 may be viewed as a generalization of [3], and we call it a strong uniqueness theorem (see the extrinsic version in [5]).

In views of [3], the whole issue is reduced to the curvature estimates. In this paper, we will derive some local curvature estimates in dimension 3 (or 2), which have their own interest from PDE point of view. Our strategy is the following. The singularities of ancient type occur naturally, once the desired estimate fails. Through the great works of Hamilton and Perelman, the structures of singularities in dimension 3 have already been well-understood nowadays. One crucial reason why Ricci flow works in dimension 3 in the classical theory is that we have Hamilton-Ivey's curvature pinching estimate, which guarantees the singularities are always nonnegative curved. Recall these estimates were proved by maximum principle. In the classical setting, this principle can only be applied on manifolds or (Ricci flow) solutions with bounded or suitable growth curvature. Remember the curvature estimate is just the goal we want to achieve. To go around this difficulty, in this paper, 
we will derive some pinching estimates of similar type, but in a purely local way (see section 2)

In this regard, let us recall the so-called pseudolocality theorem of Perelman [12]. The point we should mention here, is that the pseudolocality theorem of Perelman [12] is basically proved for compact manifolds. Since the justification of integration by parts on the whole manifold is also ultimately related to the geometry of the solution, this makes the situation very complicated (see the proof in section 10 in [12]). Actually, it is still an open problem if the pseudolocality theorem holds for any complete solutions to the Ricci flow. Recently, in $[\mathbf{2}]$, by assuming the solution has bounded curvature, the pseudolocality theorem of Perelman has been generalized to complete manifolds. As mentioned above, the key point for our strong uniqueness is just the curvature bound. In this paper, we will adopt a totally different approach.

We remark that in dimension 2, we even have a better strong uniqueness theorem, i.e. nonnegative curvature assumption can be removed (see Theorem 3.10).

The paper is organized as follows. In section 2, we derive a pure local pinching estimate for 3 dimensional Ricci flow. In section 3, we will show various local a priori curvature estimates, which may give rise to the proof of the uniqueness theorems. In section 4, we will discuss some further open problems.

Acknowledgements. The author is grateful to Professor X.P. Zhu for many stimulating discussions. The author is also thankful to Professors D. Burago, X. C. Rong and some others for the discussions on the injectivity radius assumption in the previous version of the paper. Finally, M. Simon told the author that he had similar interior estimates (provided the curvature of the solution has been controlled by $\frac{K}{t}$ ) as in our Theorem 3.1 in his habilitation thesis [15].

\section{Local pinching estimate}

Hamilton-Ivey's pinching estimate plays a substantial role in the application of the Ricci flow to the geometrization conjecture in dimension 3. As a matter of fact, this is one main reason why this theory works in this very dimension. As we mentioned in the introduction, the proof of this estimate is by maximum principle for compact or complete solutions with bounded curvature. Because the curvature bound on the whole manifold is just the goal we want to achieve, this becomes an obstacle for us. Fortunately, we find that the equation has certain good nonlinearity, which enables us to localize all the estimates.

We start with the local estimate of scalar curvature, which is dimensionally free. 
Proposition 2.1. For any $0<\delta<\frac{2}{n}$, there is $C=C(\delta, n)>0$ satisfying the following property. Suppose we have a smooth solution $g_{i j}(x, t)$ to the Ricci flow on an $n$ dimensional manifold $M$, such that for any $t \in[0, T], B_{t}\left(x_{0}, A r_{0}\right)$ are compactly contained in $M$ and assume that Ric $(x, t) \leq(n-1) r_{0}^{-2}$ for $x \in B_{t}\left(x_{0}, r_{0}\right), t \in[0, T]$ and $R \geq-K$ $(K \geq 0)$ on $B_{0}\left(x_{0}, A r_{0}\right)$ at $t=0$. Then we have

(i) $R(x, t) \geq \min \left\{-\frac{1}{\left(\frac{2}{n}-\delta\right) t+\frac{1}{K}},-\frac{C}{A r_{0}^{2}}\right\}$, if $A \geq 2$;

(ii) $R(x, t) \geq \min \left\{-\frac{1}{\left(\frac{2}{n}-\delta\right) t+\frac{n}{K}},-\frac{C}{A^{2} r_{0}^{2}}\right\}$, if $A \geq \frac{40}{3}(n-1) r_{0}^{-2} T+2$,

whenever $x \in B_{t}\left(x_{0}, \frac{3 A}{4} r_{0}\right), t \in[0, T]$.

Proof. By $[\mathbf{1 2}]$, we have

$$
\left(\frac{\partial}{\partial t}-\triangle\right) d_{t}\left(x_{0}, x\right) \geq-\frac{5(n-1)}{3} r_{0}^{-1},
$$

whenever $d_{t}\left(x, x_{0}\right)>r_{0}$, in the sense of support functions.

We divide the discussion into two cases.

Case(a): $A \geq \frac{40}{3}(n-1) r_{0}^{-2} T+2$.

We consider the function

$$
u=\varphi\left(\frac{d_{t}\left(x_{0}, \cdot\right)+\frac{5(n-1) r_{0}^{-1} t}{3}}{A r_{0}}\right) R,
$$

where $\varphi$ is a fixed smooth nonnegative non-increasing function such that $\varphi=1$ on $\left(-\infty, \frac{7}{8}\right]$, and $\varphi=0$ on $[1, \infty)$.

It is clear that

$$
\begin{aligned}
\left(\frac{\partial}{\partial t}-\triangle\right) u= & \varphi^{\prime} R \frac{1}{A r_{0}}\left[\left(\frac{\partial}{\partial t}-\triangle\right) d_{t}\left(x_{0}, x\right)+\frac{5}{3}(n-1) r_{0}^{-1}\right] \\
& -\varphi^{\prime \prime} \frac{1}{\left(A r_{0}\right)^{2}} R+2 \varphi|R i c|^{2}-2 \nabla \varphi \cdot \nabla R
\end{aligned}
$$

at smooth points of distance function.

Let $u_{\text {min }}(t)=\min _{M} u(\cdot, t)$. If $u_{\text {min }}\left(t_{0}\right) \leq 0$ and $u_{\text {min }}\left(t_{0}\right)$ is achieved at some point $x_{1}$, then $\varphi^{\prime} R\left(x_{1}, t_{0}\right) \geq 0$. Hence, by (2.1), the first term in the right hand side of $(2.2)$ is nonnegative. Now by applying the maximum principle and standard support function technique, we have for any small $\delta>0$

$$
\begin{aligned}
\left.\frac{d^{-}}{d t} u_{\min }\right|_{t=t_{0}} & :=\liminf _{\triangle t \searrow 0} \frac{u_{\min }\left(t_{0}+\Delta t\right)-u_{\min }\left(t_{0}\right)}{\triangle t} \\
& \geq \frac{2}{n} \varphi R^{2}+\frac{1}{\left(A r_{0}\right)^{2}}\left(\frac{2 \varphi^{\prime 2}}{\varphi}-\varphi^{\prime \prime}\right) R \\
& \geq\left(\frac{2}{n}-\delta\right) u_{\min }\left(t_{0}\right)^{2}+\frac{\delta}{2}\left(u_{\min }\left(t_{0}\right)^{2}-\frac{C^{2}}{\left(A r_{0}\right)^{4}}\right) .
\end{aligned}
$$


provided $u_{\min }\left(t_{0}\right) \leq 0$, where we have used $\left|\frac{2 \varphi^{\prime 2}}{\varphi}-\varphi^{\prime \prime}\right| \leq C \sqrt{\varphi}$ and Cauchy-Schwartz $\left|\frac{1}{\left(A r_{0}\right)^{2}}\left(\frac{2 \varphi^{\prime 2}}{\varphi}-\varphi^{\prime \prime}\right) R\right| \leq \frac{\delta}{2} \varphi R^{2}+\frac{C}{\left(A r_{0}\right)^{4}}$.

By integrating the inequality (2.3), we get

$$
u_{\min }(t) \geq \min \left\{-\frac{1}{\left(\frac{2}{n}-\delta\right) t+\frac{1}{K}},-\frac{C}{\left(A r_{0}\right)^{2}}\right\} .
$$

This implies

$$
R(x, t) \geq \min \left\{-\frac{1}{\left(\frac{2}{n}-\delta\right) t+\frac{1}{K}},-\frac{C(\delta)}{\left(A r_{0}\right)^{2}}\right\},
$$

whenever $x \in B_{t}\left(x_{0}, \frac{3 A}{4} r_{0}\right)$.

Case(b): $A \leq \frac{40}{3}(n-1) \operatorname{Tr}_{0}^{-2}+2$.

Consider the function $u=\varphi\left(\frac{d_{t}\left(x_{0}, \cdot\right)}{A r_{0}}\right) R$, the similar argument yields

$$
u_{\text {min }}(t) \geq \min \left\{-\frac{1}{\left(\frac{2}{n}-\delta\right) t+\frac{1}{K}},-\frac{C(\delta)}{A r_{0}^{2}}\right\} .
$$

The proof is completed.

q.e.d.

In dimension 3 , in terms of moving frames $[\mathbf{8}]$, the curvature operator, $M_{i j}=R g_{i j}-2 R_{i j}$, has the following evolution equation

$$
\frac{\partial}{\partial t} M=\triangle M+M^{2}+M^{\sharp},
$$

where $M^{\sharp}$ is the lie algebra adjoint of $M$. Let $\lambda \geq \mu \geq \nu$ be the eigenvalues of $M$, the same eigenvectors also diagnolize $M^{2}+M^{\sharp}$ with eigenvalues $\lambda^{2}+\mu \nu, \mu^{2}+\lambda \nu, \nu^{2}+\lambda \mu$. The following estimate may be viewed as a local version of Hamilton-Ivey pinching estimate.

Proposition 2.2. For any $k \in \mathbb{Z}_{+}$, there is $C_{k}$ depending only on $k$ satisfying the following property. Suppose we have a smooth solution $g_{i j}(x, t)$ to the Ricci flow on a three manifold $M$, such that for any $t \in[0, T], B_{t}\left(x_{0}, A r_{0}\right)$ are compactly contained in $M$ and assume that $\operatorname{Ric}(x, t) \leq(n-1) r_{0}^{-2}$ for $x \in B_{t}\left(x_{0}, r_{0}\right), t \in[0, T]$; and $\lambda+\mu+k \nu \geq$ $-K_{k}\left(K_{k} \geq 0\right)$ on $B_{0}\left(x_{0}, A r_{0}\right)$ at time 0 . Then we have

(i) $\lambda+\mu+k \nu \geq \min \left\{-\frac{C_{k}}{t+\frac{1}{K_{k}}},-\frac{C_{k}}{A r_{0}^{2}}\right\}$, if $A \geq 2$;

(ii) $\lambda+\mu+k \nu \geq \min \left\{-\frac{C_{k}}{t+\frac{1}{K_{k}}},-\frac{C_{k}}{A^{2} r_{0}^{2}}\right\}$, if $A \geq \frac{40(n-1) k}{3} r_{0}^{-2} T+2$,

whenever $x \in B_{t}\left(x_{0}, \frac{A}{2} r_{0}\right), t \in[0, T]$. 
Proof. We only prove the general case (i). We will argue by induction on $k \in \mathbb{Z}_{+}$to prove the estimate holds on ball of radius $\left(\frac{1}{2}+\frac{1}{2^{k_{0}+1}}\right) A r_{0}$. The $k=1$ case follows from Proposition 2.1, and radius of the ball is $\frac{3 A}{4} r_{0}$. Suppose we have proved the result for $k=k_{0} \in \mathbb{Z}_{+}$, that is to say, there is constant $C_{k_{0}}$ such that

$$
\lambda+\mu+k_{0} \nu \geq \min \left\{-\frac{C_{k_{0}}}{t+\frac{1}{K_{k_{0}}}},-\frac{C_{k_{0}}}{A r_{0}^{2}}\right\},
$$

whenever $x \in B_{t}\left(x_{0},\left(\frac{1}{2}+\frac{1}{2^{k_{0}+1}}\right) A r_{0}\right), t \in[0, T]$. We are going to prove the result for $k=k_{0}+1$ on ball of radius $\left(\frac{1}{2}+\frac{1}{2^{k_{0}+2}}\right) A r_{0}$.

Without loss of generality, we may assume $K_{1} \leq K_{2} \leq K_{3} \leq \cdots$.

Define a function $C_{k_{0}}(t):=\max \left\{\frac{C_{k_{0}}}{t+\frac{1}{K_{k_{0}}}}, \frac{C_{k_{0}}}{A r_{0}^{2}}\right\}$.

Let

$$
N_{i j}=R g_{i j}+k_{0} M_{i j}, \quad P_{i j}=\varphi\left(\frac{d_{t}\left(x, x_{0}\right)}{A r_{0}}\right)\left(R g_{i j}+k_{0} M_{i j}\right),
$$

where $\varphi$ is a smooth nonnegative decreasing function, which is 1 on $\left(-\infty, \frac{1}{2}+\frac{1}{2^{k_{0}+2}}\right]$ and 0 on $\left[\frac{1}{2}+\frac{1}{2^{k_{0}+1}}, \infty\right)$. Note that the least eigenvalue of $N_{i j}$ is $\lambda+\mu+\left(k_{0}+1\right) \nu$. Let $V$ be the corresponding (time dependent) unit eigenvector of $N_{i j}$.

By direct computation, we have

$$
\left(\frac{\partial}{\partial t}-\triangle\right) P_{i j}=-2 \nabla_{l} \varphi \nabla_{l} N_{i j}+Q_{i j}
$$

where $Q_{i j}$ satisfies

$$
\begin{aligned}
& Q(V, V)=\varphi\left(\lambda^{2}+\mu^{2}+\left(k_{0}+1\right) \nu^{2}+\mu \nu+\lambda \nu+\left(k_{0}+1\right) \lambda \mu\right) \\
& \quad+\left[\varphi^{\prime} \frac{1}{A r_{0}}\left[\left(\frac{\partial}{\partial t}-\triangle\right) d_{t}\left(x_{0}, x\right)\right]-\varphi^{\prime \prime} \frac{1}{\left(A r_{0}\right)^{2}}\right]\left(\lambda+\mu+\left(k_{0}+1\right) \nu\right) .
\end{aligned}
$$

Let

$$
u(t):=\min _{x \in M}\left(\lambda+\mu+\left(k_{0}+1\right) \nu\right) \varphi(x, t) .
$$

For fixed $t_{0} \in[0, T]$, assume $\left(\lambda+\mu+\left(k_{0}+1\right) \nu\right) \varphi\left(x_{0}^{\prime}, t_{0}\right)=u\left(t_{0}\right)<$ $-2 C_{k_{0}}\left(t_{0}\right)$. Otherwise, we have the estimate at time $t_{0}$.

Combining with (2.4), we have $\left(\lambda+\mu+\left(k_{0}-1\right) \nu\right)\left(x_{0}^{\prime}, t_{0}\right) \geq 0$. Note that $\nu\left(x_{0}^{\prime}, t_{0}\right)$ is negative, otherwise $\left(\lambda+\mu+\left(k_{0}+1\right) \nu\right)\left(x_{0}^{\prime}, t_{0}\right) \geq 0$. Hence $(\lambda+\mu) \varphi\left(x_{0}^{\prime}, t_{0}\right) \geq 0$.

We compute 


$$
\begin{aligned}
Q(V, V)\left(x_{0}^{\prime}, t_{0}\right)= & \varphi\left(\lambda^{2}+\mu^{2}+\left(k_{0}+1\right) \nu^{2}+(\lambda+\mu) \nu+\left(k_{0}+1\right) \lambda \mu\right) \\
& +\left[\frac{\varphi^{\prime}}{\varphi} \frac{1}{A r_{0}}\left(\frac{\partial}{\partial t}-\triangle\right) d_{t}\left(x_{0}, x\right)-\frac{\varphi^{\prime \prime}}{\varphi} \frac{1}{\left(A r_{0}\right)^{2}}\right] u\left(t_{0}\right) \\
= & \varphi \frac{\left(\lambda+\mu+\left(k_{0}+1\right) \nu\right)^{2}}{\left(k_{0}+1\right)}-\frac{\lambda+\mu}{k_{0}+1} \varphi\left(\lambda+\mu+\left(k_{0}+1\right) \nu\right) \\
& +\varphi\left(\lambda^{2}+\mu^{2}+\left(k_{0}+1\right) \lambda \mu\right) \\
& +\left[\frac{\varphi^{\prime}}{\varphi} \frac{1}{A r_{0}}\left(\frac{\partial}{\partial t}-\triangle\right) d_{t}\left(x_{0}, x\right)-\frac{\varphi^{\prime \prime}}{\varphi} \frac{1}{\left(A r_{0}\right)^{2}}\right] u\left(t_{0}\right) \\
= & I+I I+I I I+I V .
\end{aligned}
$$

Since $(\lambda+\mu) \varphi\left(x_{0}^{\prime}, t_{0}\right) \geq 0$ and $u\left(t_{0}\right)<0$, we have $I I \geq 0$. To deal with term $I I I$, we divide into two cases.

Case $(\alpha): \mu\left(x_{0}^{\prime}, t_{0}\right)<-\frac{\lambda\left(x_{0}^{\prime}, t_{0}\right)}{k_{0}+1}$.

By $(2.4),\left(\lambda+\mu+k_{0} \nu\right)\left(x_{0}, t_{0}\right) \geq-C_{k_{0}}\left(t_{0}\right)$, we have $-\nu\left(x_{0}^{\prime}, t_{0}\right) \leq$ $\frac{\lambda\left(x_{0}^{\prime}, t_{0}\right)}{k_{0}+1}+\frac{C_{k_{0}}\left(t_{0}\right)}{k_{0}}$. Hence at $\left(x_{0}^{\prime}, t_{0}\right)$, we have

$$
\begin{aligned}
\lambda^{2}+\mu^{2}+ & \left(k_{0}+1\right) \lambda \mu \\
& \geq \lambda^{2}+\left(\frac{\lambda}{k_{0}+1}\right)^{2}-\left(k_{0}+1\right) \lambda\left(\frac{\lambda}{k_{0}+1}+\frac{C_{k_{0}}\left(t_{0}\right)}{k_{0}}\right) \\
& \geq\left(\frac{\lambda}{k_{0}+1}\right)^{2}-\left(k_{0}+1\right) \frac{C_{k_{0}}\left(t_{0}\right)}{k_{0}} \lambda \geq-\frac{\left(k_{0}+1\right)^{4} C_{k_{0}}\left(t_{0}\right)^{2}}{4 k_{0}^{2}} .
\end{aligned}
$$

Case $(\beta): \mu\left(x_{0}^{\prime}, t_{0}\right) \geq-\frac{\lambda\left(x_{0}^{\prime}, t_{0}\right)}{k_{0}+1}$.

In this case, $\left(\lambda^{2}+\mu^{2}+\left(k_{0}+1\right) \lambda \mu\right)\left(x_{0}^{\prime}, t_{0}\right) \geq 0$ holds trivially.

Hence in either case, we have

$$
\lambda^{2}+\mu^{2}+\left(k_{0}+1\right) \lambda \mu \geq-\frac{\left(k_{0}+1\right)^{4} C_{k_{0}}^{2}\left(t_{0}\right)}{4 k_{0}^{2}} .
$$

Therefore,

$$
\begin{aligned}
Q(V, V)\left(x_{0}^{\prime}, t_{0}\right) \geq & \varphi \frac{\left(\lambda+\mu+\left(k_{0}+1\right) \nu\right)^{2}}{\left(k_{0}+1\right)}-\frac{\left(k_{0}+1\right)^{4} C_{k_{0}}\left(t_{0}\right)^{2}}{4 k_{0}^{2}} \varphi \\
& +\left[\frac{\varphi^{\prime}}{\varphi} \frac{1}{A r_{0}}\left[\left(\frac{\partial}{\partial t}-\triangle\right) d_{t}\left(x_{0}, x\right)\right]-\frac{\varphi^{\prime \prime}}{\varphi} \frac{1}{A^{2} r_{0}^{2}}\right] u\left(t_{0}\right) \\
\geq & \frac{1}{\left(k_{0}+1\right) \varphi}\left[u^{2}-\left(\frac{5(n-1) \varphi^{\prime}}{3 A r_{0}^{2}}+\frac{k_{0}+1}{A^{2} r_{0}^{2}} \varphi^{\prime \prime}\right) u\right] \\
& -\frac{\left(k_{0}+1\right)^{4} C_{k_{0}}^{2}\left(t_{0}\right)}{4 k_{0}^{2}} .
\end{aligned}
$$


Since $\left|\varphi^{\prime}\right| \leq C 2^{k_{0}},\left|\varphi^{\prime \prime}\right|+\frac{\varphi^{\prime 2}}{\varphi} \leq C 2^{2 k_{0}}$, by applying maximum principle, we have

$$
\begin{aligned}
\left.\frac{d^{-}}{d t}\right|_{t=t_{0}} u & \geq Q(V, V)\left(x_{0}^{\prime}, t_{0}\right)+\frac{2}{\left(A r_{0}\right)^{2}} \frac{\varphi^{\prime 2}}{\varphi^{2}} u\left(t_{0}\right) \\
& \geq \frac{1}{2\left(k_{0}+1\right)} u^{2}
\end{aligned}
$$

provided $|u|\left(t_{0}\right) \geq \max \left\{C C_{k_{0}}\left(t_{0}\right) k_{0}^{\frac{3}{2}}, C \frac{2^{2 k_{0}} k_{0}}{A r_{0}^{2}}\right\}$, where $C$ is some universal constant. By integrating the above differential inequality, we get estimate:

$$
u(t) \geq \min \left\{\frac{1}{\frac{1}{u(0)}-\frac{t}{2\left(k_{0}+1\right)}},-C C_{k_{0}}(t) k_{0} \frac{3}{2},-C \frac{2^{2 k_{0}} k_{0}}{A r_{0}^{2}}\right\} .
$$

By the definition of $C_{k_{0}}(t)$, noting $-K_{k_{0}} \geq-K_{k_{0}+1}$, clearly, there is a $C_{k_{0}+1}$ such that

$$
u(t) \geq \min \left\{-\frac{C_{k_{0}+1}}{t+\frac{1}{K_{k_{0}+1}}},-\frac{C_{k_{0}+1}}{A r_{0}^{2}}\right\} .
$$

The proof of case (ii) is similar. We use cut-off function

$$
\varphi\left(\frac{d_{t}\left(x_{0}, \cdot\right)+\frac{5(n-1) r_{0}^{-1} t}{3}}{A r_{0}}\right)
$$

where $\varphi$ is a suitably chosen function which depends on $k_{0}$ in the inductive step.

q.e.d.

We remark that by following the constants in the proof, the constant $C_{k}$ may be chosen to be $C k^{C k}$ for some universal constant $C$. The factor $\frac{1}{2}$ in the radius $\frac{1}{2} A r_{0}$ is not important, it may be replaced by any constant in $(0,1)$.

Corollary 2.3. Suppose we have a complete smooth solution $g_{i j}(x, t)$ to the Ricci flow on $M \times[0, T]$, then whenever $t \in[0, T]$ we have

(i) if $R \geq-K$ for $0 \leq K \leq \infty$ at $t=0$, then

$$
R(\cdot, t) \geq-\frac{n}{2 t+\frac{n}{K}}
$$

(ii) if $\operatorname{dim} M=3$, then for any $k>0$, there is $C_{k}>0$ depending only on $k$ such that if at $t=0, \lambda+\mu+k \nu \geq-K_{k}$ for some $0 \leq K_{k} \leq \infty$, then

$$
\lambda+\mu+k \nu \geq-\frac{C_{k}}{t+\frac{1}{K_{k}}}
$$


Proof. For fixed $x_{0} \in M$, since the solution is smooth, there is a small $r_{0}>0$ such that whenever $t \in[0, T], x \in B_{t}\left(x_{0}, r_{0}\right)$, we have

$$
|R m|(x, t) \leq r_{0}^{-2} \text {. }
$$

For the proof of (i), let $A \rightarrow \infty, \delta \rightarrow 0$ in the Proposition 2.1, we get the desired estimate. Case (ii) follows from Proposition 2.2 by letting $A \rightarrow \infty$.

q.e.d.

In particular, in dimension 3 , if the sectional curvature is nonnegative at $t=0$, then this property is preserved for $t>0$ for any complete solutions.

Furthermore, for complete ancient solution, for any fixed $t \in(-\infty, 0]$, by Corollary 2.3 (ii), we have $(\lambda+\mu+k \nu)(t) \geq-\frac{C_{k}}{t-(-T)}$ for any $T>0$. Since $C_{k}$ depends only on $k$, we have $(\lambda+\mu+k \nu)(t) \geq 0$ for any $k \in \mathbb{Z}_{+}$. This implies $\nu \geq 0$, i.e. the sectional curvature is nonnegative.

Corollary 2.4. Any ancient smooth complete solution to the Ricci flow (not necessarily having bounded curvature) on three manifold must have nonnegative sectional curvature.

Corollary 2.5. Any ancient smooth complete solution to the Ricci flow (not necessarily having bounded curvature) must have nonnegative scalar curvature.

\section{A priori estimates}

3.1. We will prove the following preliminary interior estimate, which holds for any dimension.

Theorem 3.1. There is a constant $C=C(n)$ with the following property. Suppose we have a smooth solution to the Ricci flow $\left(g_{i j}\right)_{t}=$ $-2 R_{i j}, 0 \leq t \leq T$, on an $n$-manifold $M$ such that $B_{t}\left(x_{0}, r_{0}\right), 0 \leq t \leq T$, is compactly contained in $M$ and

(i) $|R m| \leq r_{0}^{-2}$ on $B_{0}\left(x_{0}, r_{0}\right)$ at $t=0$;

(ii) $|R m|(x, t) \leq \frac{K}{t}$ where $K \geq 1, d_{t}(x, t)=\operatorname{dist}_{t}\left(x_{0}, x\right)<r_{0}$, whenever $0 \leq t \leq T$.

Then we have

$$
|R m|(x, t) \leq e^{C K}\left(r_{0}-d_{t}\left(x_{0}, x\right)\right)^{-2}
$$

whenever $0 \leq t \leq T, d_{t}(x, t)=\operatorname{dist}_{t}\left(x_{0}, x\right)<r_{0}$.

Proof. By scaling, we may assume $r_{0}=1$.

Since the result holds trivially by assumption when $t \geq 1$. Without loss of generality, we may assume $T \leq 1$.

We argue by contradiction. Suppose we have a sequence of $\delta \rightarrow 0$, and a sequence of solutions satisfying the assumptions in Theorem 3.1. But 
$|R m|\left(x_{1}, t_{1}\right)>e^{\frac{K}{\delta}} \varepsilon^{-2}$ holds for some point $\left(x_{1}, t_{1}\right), d_{t_{1}}\left(x_{1}, x_{0}\right)<1-\varepsilon$, $t_{1} \in[0, T]$.

For any fixed $B \geq 1$, by a point-picking technique of Perelman [12], we can choose another point $(\bar{x}, \bar{t}), \bar{x} \in B_{\bar{t}}\left(x_{0}, 1-\frac{\varepsilon}{2}\right), \bar{t} \in\left(0, t_{1}\right]$ such that $\bar{Q}=|R m|(\bar{x}, \bar{t}) \geq e^{\frac{K}{\delta}} \varepsilon^{-2}$ and

$$
|R m|(x, t) \leq 2 \bar{Q}
$$

whenever $d_{t}\left(x_{0}, x\right) \leq d_{\bar{t}}\left(\bar{x}, x_{0}\right)+10 B K \bar{Q}^{-\frac{1}{2}}, 0 \leq t \leq \bar{t}$.

At the end of the proof, it turns out that we only need to choose $B=2 \frac{e^{C(n) K}-1}{K}$.

Actually $(\bar{x}, \bar{t})$ can be constructed as the limit of a finite sequence $\left(x_{i}, t_{i}\right)$ satisfying $0 \leq t_{k} \leq t_{k-1}, d_{t_{k}}\left(x_{0}, x_{k}\right) \leq d_{t_{k-1}}\left(x_{0}, x_{k-1}\right)+10 B K$ $|R m|\left(x_{k-1}, t_{k-1}\right)^{-\frac{1}{2}},|R m|\left(x_{k}, t_{k}\right) \geq 2|R m|\left(x_{k-1}, t_{k-1}\right)$. Since

$$
\begin{aligned}
|R m|\left(x_{k}, t_{k}\right) & \geq 2^{k-1}|R m|\left(x_{1}, t_{1}\right) \geq 2^{k-1} e^{\frac{K}{\delta}} \varepsilon^{-2}, \text { we have } \\
d_{t_{k}}\left(x_{0}, x_{k}\right) \leq & d_{t_{1}}\left(x_{0}, x_{1}\right)+10 B K \sum_{i=1}^{\infty}\left(2^{i-1}|R m|\left(x_{1}, t_{1}\right)\right)^{-\frac{1}{2}} \\
& \leq 1-\varepsilon+40 B K e^{-\frac{K}{2 \delta}} \varepsilon \leq 1-\frac{\varepsilon}{2} .
\end{aligned}
$$

Clearly, if we choose $B=2 \frac{e^{C(n) K}-1}{K}$, the last inequality is guaranteed by $e^{\left(C(n)-\frac{1}{2 \delta}\right) K} \leq \frac{1}{160}$, which holds trivially since $K \geq 1$ and $\delta \rightarrow 0$. Since the solution is smooth, this sequence must be finite and the last element is what we want.

From this construction, we know $d_{\bar{t}}\left(\bar{x}, x_{0}\right)+10 B K \bar{Q}^{-\frac{1}{2}} \leq 1-\frac{\varepsilon}{2}$.

We denote by $C(n)$ various universal big constants depending only upon the dimension. In the following argument, it may vary line by line.

Now let $\varphi$ be a fixed smooth nonnegative non-increasing cut-off function such that $\varphi=1$ on $\left(-\infty, d_{\bar{t}}\left(\bar{x}, x_{0}\right)+B K \bar{Q}^{-\frac{1}{2}}\right], \varphi=0$ on $\left[d_{\bar{t}}\left(\bar{x}, x_{0}\right)\right.$ $\left.+10 B K \bar{Q}^{-\frac{1}{2}}, \infty\right)$. Clearly, we have

$$
\left|\varphi^{\prime}\right| \leq C \frac{\bar{Q}^{\frac{1}{2}}}{B K},\left|\varphi^{\prime \prime}\right|+\frac{\left|\varphi^{\prime}\right|^{2}}{\varphi} \leq C \frac{\bar{Q}}{(B K)^{2}} .
$$

Consider the function $u=\varphi\left(d_{t}\left(x_{0}, x\right)\right)|R m|(x, t)^{2}$, it is clear that

$$
\begin{aligned}
\left(\frac{\partial}{\partial t}-\triangle\right) u & \leq \varphi^{\prime}|R m|^{2}\left(\frac{\partial}{\partial t}-\triangle\right) d_{t}\left(x_{0}, x\right)-2 \varphi|\nabla R m|^{2} \\
& -\varphi^{\prime \prime}|R m|^{2}+C(n) \varphi|R m|^{3}-2 \nabla \varphi \cdot \nabla|R m|^{2} .
\end{aligned}
$$

Since by (3.1), $\left(\frac{\partial}{\partial t}-\triangle\right) d_{t}\left(x_{0}, x\right) \geq-C(n) \bar{Q}^{\frac{1}{2}}$ whenever $\bar{Q}^{-\frac{1}{2}}<$ $d_{t}\left(x_{0}, x\right)$. Then by the maximum principle, and $(3.1)(3.2)$, it is clear 
that at the maximum point,

$$
\begin{aligned}
\frac{d^{+}}{d t} u_{\max } & \leq \frac{C(n)}{B K}|R m|^{2} \bar{Q}+C \varphi|R m|^{3} \\
& \leq \frac{C(n)}{B K} \bar{Q}^{3}+C(n) \bar{Q} u_{\max }(t) .
\end{aligned}
$$

Integrating this inequality, noting $u_{\max }(0) \leq 1$ by assumption, we get

$$
\left.e^{-C(n) \bar{Q} t} u_{\max }(t)\right|_{t=0} ^{t=\bar{t}} \leq-\left.\frac{\bar{Q}^{2}}{B K} e^{-C(n) \bar{Q} t}\right|_{t=0} ^{t=\bar{t}},
$$

and

$$
u_{\max }(\bar{t}) \leq e^{C(n) \bar{Q} \bar{t}}+\frac{1}{B K}\left(e^{C(n) \bar{Q} \bar{t}}-1\right) \bar{Q}^{2} .
$$

Since $u_{\max }(\bar{t}) \geq u(\bar{x}, \bar{t})=\bar{Q}^{2}$, and $\bar{Q} \bar{t} \leq K$, we have

$$
\left(1-\frac{e^{C(n) K}-1}{B K}\right) \bar{Q}^{2} \leq e^{C(n) K} .
$$

Therefore, if we choose $B=\frac{2\left(e^{C(n) K}-1\right)}{K}$, then we have

$$
\bar{Q} \leq e^{C(n) K}
$$

which is a contradiction with $\bar{Q} \geq e^{\frac{K}{\delta}} \varepsilon^{-2}$ as $\delta \rightarrow 0$.

This completes the proof of the Theorem 3.1.

q.e.d.

Corollary 3.2. Suppose we have a smooth solution to the Ricci flow $\left(g_{i j}\right)_{t}=-2 R_{i j}, 0 \leq t \leq T$, such that at $t=0$ we have $|R m| \leq r_{0}^{-2}$ on $B_{0}\left(x_{0}, r_{0}\right)$; and

$$
|R m|(x, t) \leq \frac{K}{t}
$$

whenever $0<t \leq T, d_{0}(x, t)=$ dist $_{0}\left(x_{0}, x\right)<r_{0}$. Here we assume $B_{0}\left(x_{0}, r_{0}\right)$ is compactly contained in the manifold $M$. Then there is a constant $C$ depending only on the dimension such that the following estimate

$$
|R m|(x, t) \leq e^{C K}\left(r_{0}-d_{0}\left(x_{0}, x\right)\right)^{-2}
$$

holds for all $(x, t) \in B_{0}\left(x_{0}, r_{0}\right) \times[0, T]$.

Proof. By [12], for any fixed $p \in B_{0}\left(x_{0}, r_{0}\right)$, as long as the minimal geodesic $\gamma_{t}$ at time $t \in\left[0, r_{0}^{2}\right]$ connecting $p$ and $x_{0}$ lies in $B_{0}\left(x_{0}, r_{0}\right)$, we have

$$
\frac{d}{d t} d_{t}\left(x_{0}, p\right) \geq-C(n) \sqrt{\frac{K}{t}}
$$

For any fixed $p \in B_{0}\left(x_{0}, r_{0}\right)$, let $\left[0, T^{\prime}\right)$ be the largest interval such that any minimal geodesic $\gamma_{t}$ at time $t \in\left[0, T^{\prime}\right]$ connecting $x_{0}$ and $p$ lies in $B_{0}\left(x_{0}, r_{0}\right)$ entirely. By integrating the above inequality, we get

$$
d_{0}\left(x_{0}, p\right) \leq d_{t}\left(x_{0}, p\right)+C(n) \sqrt{K} \sqrt{T^{\prime}} .
$$


This implies $B_{t}\left(x_{0}, \frac{r_{0}}{4}\right) \subset B_{0}\left(x_{0}, \frac{r_{0}}{2}\right)$, for any $t \in\left[0, \frac{r_{0}^{2}}{C(n) K}\right]$. By applying Theorem 3.1 with $T=\frac{r_{0}^{2}}{C(n) K}<\left(\frac{r_{0}}{4}\right)^{2}$, there is a constant $C(n)$ depending only on the dimension, such that $|R m| \leq e^{C(n) K} r_{0}^{-2}$ whenever $0<t<\frac{r_{0}^{2}}{C(n) K}, d_{t}(x, t)=\operatorname{dist}_{t}\left(x_{0}, x\right)<\frac{1}{8} r_{0}$. On the other hand, for $d_{0}\left(x_{0}, x\right)<r_{0}$ and $t \in\left[\frac{r_{0}^{2}}{C(n) K}, r_{0}^{2}\right]$, by assumption, we always have

$$
|R m|(x, t) \leq \frac{K}{t} \leq e^{C(n) K} r_{0}^{-2} .
$$

This in particular implies $|R m|\left(x_{0}, t\right) \leq e^{C(n) K} r_{0}^{-2}$, for any $t \in[0, T]$.

For any $x \in B_{0}\left(x_{0}, r_{0}\right)$, apply the above estimate on ball $B_{0}\left(x, r_{0}-\right.$ $\left.d_{0}\left(x_{0}, x\right)\right)$ again, we know $|R m|(x, t) \leq e^{C(n) K}\left(r_{0}-d_{0}\left(x_{0}, x\right)\right)^{-2}$ for any $t \in\left[0,\left(r_{0}-d_{0}\left(x_{0}, x\right)\right)^{2}\right]$. For $t>\left(r_{0}-d_{0}\left(x_{0}, x\right)\right)^{2}$, we have

$$
|R m|(x, t) \leq \frac{K}{t} \leq \frac{K}{\left(r_{0}-d_{0}\left(x_{0}, x\right)\right)^{2}} \leq e^{C(n) K}\left(r_{0}-d_{0}\left(x_{0}, x\right)\right)^{-2} .
$$

The proof is completed.

q.e.d.

3.2. We say that a solution to the Ricci flow is ancient if it exists at least on a half infinite time interval $(-\infty, T)$ for some finite number $T$. Ancient solution appears naturally in the blow up argument of singularity analysis of Ricci flow. The following lemma will be used frequently in the a priori estimates of this section.

Lemma 3.3. Let $g_{i j}(x, t), t \in(-\infty, T)$ be a complete smooth nonflat ancient solution to the Ricci flow on an $n$-dimensional manifold $M$, with bounded and nonnegative curvature operator. Then for any $t \in(-\infty, T)$, the asymptotic volume ratio satisfies

$$
\nu_{M}(t):=\lim _{r \rightarrow \infty} \frac{\operatorname{vol}_{t}\left(B_{t}(x, r)\right)}{r^{n}}=0 .
$$

This lemma was proved by $[\mathbf{1 2}]$.

Theorem 3.4. For any $C>0$, there exists $K>0$ with the following properties. Suppose we have a three dimensional smooth complete solution to the Ricci flow $\left(g_{i j}\right)_{t}=-2 R_{i j}, 0 \leq t \leq T$, on a manifold $M$, and assume that at $t=0$ we have $|R m|(x, 0) \leq r_{0}^{-2}$ on $B_{0}\left(x_{0}, r_{0}\right)$, and $R(x, 0) \geq-r_{0}^{-2}$ on $M$. If $g_{i j}(x, t) \geq \frac{1}{C} g_{i j}(x, 0)$ for $x \in B_{0}\left(x_{0}, r_{0}\right)$, $t \in\left[0, r_{0}^{2}\right]$, then we have

$$
|R m|(x, t) \leq 2 r_{0}^{-2}
$$

whenever $0 \leq t \leq \min \left\{\frac{1}{K} r_{0}^{2}, T\right\}$, dist $t_{t}\left(x_{0}, x\right)<\frac{1}{K} r_{0}$.

Proof. By scaling, let $r_{0}=1$. By assumption $g_{i j}(x, t) \geq \frac{1}{C} g_{i j}(x, 0)$, we have

$$
B_{t}\left(x_{0}, \frac{1}{\sqrt{C}}\right) \subseteq B_{0}\left(x_{0}, 1\right)
$$


Let $T_{0}$ be the largest time such that $|R m|(x, t) \leq 2$ whenever $x \in$ $B_{t}\left(x_{0}, \frac{1}{2 \sqrt{C}}\right), t \in\left[0, T_{0}\right]$. We may assume $T_{0}<\min \{1, T\}$. Otherwise, there is nothing to show. Hence there is a $\left(x_{1}, t_{1}\right)$ such that $|R m|\left(x_{1}, t_{1}\right)=2, x_{1} \in B_{t_{1}}\left(x_{0}, \frac{1}{2 \sqrt{C}}\right)$ and $t_{1} \leq T_{0}$.

In the following arguments, we use $\bar{C}$ to denote various constants depending only on $C$. By using Corollary 2.3, we know

$$
R(x, t) \geq-\bar{C},
$$

on $M \times\left[0, T_{0}\right]$. By evolution equation of the volume element $\frac{d}{d t} \log \operatorname{det}(g)$ $=-R$, this gives $\frac{\operatorname{det}(g)(t)}{\operatorname{det}(g)(0)} \leq \bar{C}$. Combining with the assumption $g(t) \geq$ $\frac{1}{C} g(0)$, we have

$$
\frac{1}{\bar{C}} g(0) \leq g(t) \leq \bar{C} g(0)
$$

on $B_{0}\left(x_{0}, 1\right) \times\left[0, T_{0}\right]$.

Since the curvature on $B_{0}\left(x_{0}, 1\right)$ of the initial metric $g$ is bounded by 1 , the exponential map (for the initial metric) at $x_{0}$ is a local diffeomorphism from $B(0,1) \subset T_{P} M$ to the geodesic ball $B_{0}\left(x_{0}, 1\right)$, and such that $(\sin 1) \delta_{i j} \leq \exp ^{*} g_{i j}(x, 0) \leq(\sinh 1) \delta_{i j}$ on $B(0,1)$. By the above estimate (3.4), we have

$$
\frac{1}{\bar{C}} \delta_{i j} \leq \exp ^{*} g_{i j}(x, t) \leq \bar{C} \delta_{i j}
$$

on $B(0,1) \times\left[0, T_{0}\right]$. Let $\bar{g}(\cdot, t)=\exp ^{*} g(\cdot, t)$, then $\bar{g}(\cdot, t)$ is a solution to the Ricci flow on the Euclidean ball $B(0,1)$, moreover it is $\kappa$-noncollapsed for some $\kappa=\kappa(C)$ for all scales less than 1 by (3.5).

Now we claim that there is a constant $K_{0}>0$ depending only on $C$ such that

$$
|R m|(x, t) \leq K_{0}
$$

as $x \in B_{t}\left(0, \frac{3}{4 \sqrt{C}}\right), t \in\left[0, T_{0}\right]$.

Actually, suppose (3.6) is not true, then there is a $\left(x_{2}, t_{2}\right)$ such that $|R m|\left(x_{2}, t_{2}\right) \geq K_{1} \rightarrow \infty, x_{2} \in B_{t_{2}}\left(0, \frac{3}{4 \sqrt{C}}\right), 0<t_{2} \leq T_{0}$. Now we can choose another point $(\bar{x}, \bar{t})$ so that $\bar{Q}=|R m|(\bar{x}, \bar{t}) \geq K_{1}, \frac{1}{2 \sqrt{C}} \leq$ $d_{\bar{t}}(\bar{x}, 0) \leq \frac{7}{8 \sqrt{C}}, 0<\bar{t} \leq t_{2}$, and

$$
|R m|(x, t) \leq 4 \bar{Q}
$$

for all $d_{t}(0, x) \leq d_{\bar{t}}(0, \bar{x})+K_{1}^{\frac{1}{4}} \bar{Q}^{-\frac{1}{2}}, 0 \leq t \leq \bar{t}$.

Since $K \rightarrow \infty$, we know

$$
d_{\bar{t}}(0, \bar{x})+K_{1}^{\frac{1}{4}} \bar{Q}^{-\frac{1}{2}} \leq \frac{15}{16 \sqrt{C}} .
$$


Moreover by [12] and (3.7), it follows

$$
\frac{d}{d t} d_{t}(0, \bar{x}) \geq-\bar{C} \sqrt{\bar{Q}}
$$

whenever $d_{t}(0, \bar{x}) \leq d_{\bar{t}}(0, \bar{x})+\frac{1}{2} K_{1}^{\frac{1}{4}} \bar{Q}^{-\frac{1}{2}}$. By integrating this inequality, it is not hard to see $d_{t}(0, \bar{x}) \leq d_{\bar{t}}(0, \bar{x})+\bar{C} K_{1}^{\frac{1}{8}} \bar{Q}^{-\frac{1}{2}}$ whenever $0 \leq \bar{Q}(\bar{t}-$ $t) \leq \min \left\{K^{\frac{1}{8}}, \frac{\bar{Q} \bar{t}}{2}\right\}$. Hence, if $d_{t}(\bar{x}, x) \leq K_{1}^{\frac{1}{8}} \bar{Q}^{-\frac{1}{2}}, 0 \leq \bar{Q}(\bar{t}-t) \leq$ $\min \left\{K_{1}^{\frac{1}{8}}, \frac{\bar{Q} \bar{t}}{2}\right\}$, we have $d_{t}(0, x) \leq d_{\bar{t}}(0, \bar{x})+\bar{C} K_{1}^{\frac{1}{8}} \bar{Q}^{-\frac{1}{2}}$. By (3.7), this gives

$$
|R m|(x, t) \leq 4 \bar{Q}, \quad d_{t}(0, x) \leq \frac{15}{\sqrt{16 C}},
$$

for $x \in B_{t}\left(\bar{x}, K^{\frac{1}{8}} \bar{Q}^{-\frac{1}{2}}\right)$, and $0 \leq \bar{Q}(\bar{t}-t) \leq \min \left\{K^{\frac{1}{8}}, \frac{\bar{Q} \bar{t}}{2}\right\}$.

Recall in this region, we always have (3.5) because of (3.9) and (3.3).

Next, we will show

$$
\bar{Q} \bar{t} \rightarrow \infty
$$

which guarantees that the limit, which will be extracted from a subsequence of the rescaled solutions around $(\bar{x}, \bar{t})$, is ancient.

Let $\varphi$ be a fixed smooth nonnegative non-increasing cut-off function such that $\varphi=1$ on $\left(-\infty, d_{\bar{t}}(0, \bar{x})\right], \varphi=0$ on $\left[d_{\bar{t}}(0, \bar{x})+K_{1}^{\frac{1}{4}} \bar{Q}^{-\frac{1}{2}}, \infty\right)$.

Consider $u=\varphi\left(d_{t}(0, x)\right)|R m|(x, t)^{2}$, by applying the maximum principle as before, we have

$$
\frac{d^{+}}{d t} u_{\max } \leq \bar{C} K_{1}^{-\frac{1}{4}} \bar{Q}^{3}+\bar{C} \bar{Q} u_{\max }(t) .
$$

which gives

$$
\bar{Q}^{2} \leq e^{\bar{C} \bar{Q} \bar{t}}+\bar{Q}^{2} \bar{C} K^{-\frac{1}{4}}\left(e^{\bar{C} \bar{Q} \bar{t}}-1\right) .
$$

This implies $\bar{Q} \bar{t} \rightarrow \infty$ because $\bar{Q} \geq K_{1} \rightarrow \infty$.

So by rescaling the solution around the point $(\bar{x}, \bar{t})$ with the factor $\bar{Q}$ and shifting the time $\bar{t}$ to 0 , and using Hamilton's compactness theorem and taking convergent subsequence, we get a smooth limit. Note the curvature norm at the new origin is 1 . This limit is a nontrivial smooth complete ancient solution to the Ricci flow with bounded curvature $(\leq 4)$. By Corollary 2.4, this limit has nonnegative curvature. But (3.5) indicates the asymptotic volume ratio of the limit is strictly positive, which is a contradiction with Lemma 3.3. So we have proved the claim (3.6).

Let $\varphi$ be a fixed smooth nonnegative non-increasing cut-off function such that $\varphi=1$ on $\left(-\infty, \frac{1}{2 \sqrt{C}}\right], \varphi=0$ on $\left[\frac{3}{4 \sqrt{C}}, \infty\right)$. Consider the 
function

$$
u(x, t)=\varphi\left(d_{t}(0, x)\right)|R m|^{2}(x, t),
$$

and by (3.6) and maximum principle, we obtain

$$
\frac{d^{+}}{d t} u_{\max } \leq \bar{C}
$$

whenever $0 \leq t \leq T_{0}$. Recall we have $|R m|\left(x_{1}, t_{1}\right)=2$ for some $x_{1} \in$ $B_{t_{1}}\left(x_{0}, \frac{1}{2 \sqrt{C}}\right)$ and $t_{1} \leq T_{0}$. This gives $2 \leq u_{\max }\left(t_{1}\right) \leq 1+\bar{C} t_{1}$. Hence $T_{0} \geq \frac{1}{C}$. The proof is completed.

q.e.d.

Corollary 3.5. For any $C, K_{0}>0$, there exists a constant $K$ satisfying the following property. Suppose we have a three dimensional smooth complete solution to the Ricci flow $\left(g_{i j}\right)_{t}=-2 R_{i j}, 0 \leq t \leq T$, on a manifold $M$, and assume that at $t=0$ we have $|R m|(\cdot, 0) \leq K_{0}$ on $M$. If $g_{i j}(\cdot, t) \geq \frac{1}{C} g_{i j}(\cdot, 0)$ on $M \times[0, T]$, then we have

$$
|R m|(\cdot, t) \leq K
$$

for all $0 \leq t \leq T$.

Proof. First of all, by Theorem 3.4 we know there is a constant $T_{0}$ depending only on $K_{0}$ and $C$ such that

$$
|R m|(\cdot, t) \leq 2 K_{0}
$$

for $0 \leq t \leq \min \left\{T_{0}, T\right\}$ for some $T_{0}$. Without loss of generality, we assume $T_{0}<T$. By Corollary 2.3 and assumption, we have

$$
\frac{1}{\bar{C}} g(\cdot, 0) \leq g(\cdot, t) \leq \bar{C} g(\cdot, 0)
$$

on $M \times[0, T]$.

To prove the result, we will argue by contradiction. Suppose there is a point $\left(x_{1}, t_{1}\right)$ such that $|R m|\left(x_{1}, t_{1}\right) \geq K \rightarrow \infty$. We can choose another point $(\bar{x}, \bar{t})$ such that $\bar{Q}=|R m|(\bar{x}, \bar{t}) \geq K, \bar{t} \leq t_{1}$ and $|R m|(x, t) \leq 4 \bar{Q}$, for all $d_{t}(x, \bar{x}) \leq K^{\frac{1}{4}} \bar{Q}^{-\frac{1}{2}}$.

Otherwise, we obtain a sequence of points $\left(x_{k}, t_{k}\right)$, such that

$$
\begin{gathered}
t_{1} \geq t_{2} \geq \cdots,|R m|\left(x_{k}, t_{k}\right) \geq 4^{k-1}|R m|\left(x_{1}, t_{1}\right), \text { and } \\
d_{t_{k}}\left(x_{k}, x_{1}\right) \leq \bar{C} K^{\frac{1}{4}} \sum\left(4^{k-1}|R m|\left(x_{1}, t_{1}\right)\right)^{-\frac{1}{2}} \leq \bar{C} .
\end{gathered}
$$

Since $d_{t_{k}}\left(x_{k}, x_{1}\right) \geq \frac{1}{\bar{C}} d_{0}\left(x_{k}, x_{1}\right)$, and the solution is smooth, this procedure has to stop after a finite number of steps. Now we pull back the solution locally by using the exponential map (of the initial metric) at $\bar{x}$ to the Euclidean ball of some fixed radius as before, and notice $K^{\frac{1}{4}} \bar{Q}^{-\frac{1}{2}} \leq \bar{C} K^{-\frac{1}{4}} \ll 1$ and (3.12). Then we can rescale the solutions by the factor $\bar{Q}$ around $(\bar{x}, \bar{t})$ and extract a convergent subsequence. By (3.11), the limit is ancient. The curvature (of the limit) is bounded (by 4). So by Corollary 2.4, the limit has nonnegative sectional curvature. 
It is clear that by (3.12) and the construction, the limit has maximal volume growth. So this is a contradiction with Lemma 3.3. The proof is completed.

q.e.d.

3.3.

Theorem 3.6. For any $v_{0}>0$, there is $K>0$ depending only on $v_{0}$ with the following properties. Let $(M, g(x, 0))$ be a compete smooth 3-dimensional Riemannian manifold with nonnegative sectional curvature, $x_{0} \in M$ be a fixed point satisfying $|R m| \leq r_{0}^{-2}$ on $B_{0}\left(x_{0}, r_{0}\right)$ and $\left.\operatorname{vol}_{0}\left(B_{0}\left(x_{0}, r_{0}\right)\right)\right) \geq v_{0} r_{0}^{3}$, for some $r_{0}>0$.

Let $g(x, t), t \in[0, T]$ be a smooth complete solution to the Ricci flow with $g(x, 0)$ as initial metric. Then we have

$$
|R m|(x, t) \leq 2 r_{0}^{-2}
$$

for all $x \in B_{t}\left(x_{0}, \frac{r_{0}}{2}\right), 0 \leq t \leq \min \left\{T, \frac{1}{K} r_{0}^{2}\right\}$.

Proof. First of all, by Corollary 2.3, for any $k>0$, there is $C_{k}>0$ depending only on $k$ such that if at $t=0, \lambda+\mu+k \nu \geq-K_{k}$ for some $0 \leq K_{k} \leq \infty$, then for $t>0$, we have

$$
\lambda+\mu+k \nu \geq-\frac{C_{k}}{t+\frac{1}{K_{k}}} .
$$

In our case, $\nu \geq 0$ at $t=0$, so we can choose $K_{k}=0$ for all $k>0$. Therefore, $\lambda+\mu+k \nu \geq 0$ for $t>0$, for any $k>0$. This implies $\nu \geq 0$, i.e. curvatures are still nonnegative for $t>0$.

By scaling, we assume $r_{0}=2$.

We imitate the proof of Theorem 3.4. For the fixed $x_{0} \in M$, let $T_{0}$ be the largest time such that $|R m(x, t)| \leq \frac{1}{2}$ for all $x \in B_{t}\left(x_{0}, 1\right)$ and $t \in\left[0, T_{0}\right]$. Recall by assumption $|R m(x, 0)| \leq \frac{1}{4}$ on $B_{0}\left(x_{0}, 2\right)$. Without loss of generality, we assume $T_{0}<T$. Then there is $\left(x_{1}, t_{1}\right)$ such that $t_{1} \leq T_{0}, x_{1} \in B_{t_{1}}\left(x_{0}, 1\right),|R m|\left(x_{1}, t_{1}\right)=\frac{1}{2}$. Our purpose is to estimate $T_{0}$ from below by a positive constant depending only on $v_{0}$.

Now we claim for fixed $r>1$ there is a $B>0$ depending on $\frac{v_{0}}{r^{3}}$, such that

$$
|R m(x, t)| \leq B+B t^{-1}
$$

whenever $x \in B_{t}\left(x_{0}, \frac{r}{4}\right)$ and $t \in\left[0, T_{0}\right]$.

We will argue by contradiction. Actually, suppose (3.13) does not hold, then there is a sequence of solutions such that there is some $\left(x_{1}, t_{1}\right)$, $x_{1} \in B_{t_{1}}\left(x_{0}, \frac{r}{4}\right)$ and $t_{1} \in\left[0, T_{0}\right]$ satisfying $\left|R m\left(x_{1}, t_{1}\right)\right| \geq B+B t_{1}^{-1}$ with $B \rightarrow \infty$. By a point-picking technique of Perelman [12] (Claim 1 and Claim 2 in Theorem 10 in $[\mathbf{1 2}])$, we can choose another $(\bar{x}, \bar{t})$, with $\bar{Q}=|R m|(\bar{x}, \bar{t}) \geq \frac{B}{t}$ such that

$$
|R m|(x, t) \leq 4 \bar{Q}
$$


for all $d_{t}(x, \bar{x}) \leq A^{\frac{1}{2}} \bar{Q}^{-\frac{1}{2}}, \bar{t}-A \bar{Q}^{-1} \leq t \leq \bar{t}$, where $A$ tends to infinity with $B$.

Note that we have $\operatorname{vol}_{t}\left(B_{t}\left(x_{0}, r\right)\right) \geq \frac{v_{0}}{C r^{3}} r^{3}$, for $t \in\left[0, T_{0}\right]$. Since the curvature is nonnegative for the solution, by volume comparison theorem, the solution is $\kappa=\kappa\left(\frac{v_{0}}{r^{3}}\right)$ non-collapsed on $B_{t}\left(x_{0}, r\right)$, for $t \leq T_{0}$. So we can rescale the solution around $(\bar{x}, \bar{t})$ and extract a subsequence, finally obtain a nontrivial ancient smooth complete solution to the Ricci flow, which has maximal volume growth and bounded nonnegative curvature. This is a contradiction with Lemma 3.3. Therefore the claim (3.13) is proved.

Now by choosing $r=8$ and applying Theorem 3.1, we have $|R m(x, t)|$ $\leq$ Const on $B_{t}\left(x_{0}, \frac{3}{2}\right), t \in\left[0, T_{0}\right]$. Here the constant depends only on $v_{0}$.

Consider the evolution equation of $\varphi\left(d_{t}\left(x_{0}, x\right)\right)|R m|(x, t)$, where $\varphi$ be a smooth nonnegative decreasing function which is 1 in $(-\infty, 1]$ and 0 in $\left[\frac{3}{2}, \infty\right)$. As in the proof of Theorem 3.4, by applying maximum principle to the equation of $\varphi\left(d_{t}\left(x_{0}, x\right)\right)|R m|(x, t)$, we conclude with $T_{0} \geq \min \left\{T, \frac{1}{C}\right\}$. This completes the proof.

q.e.d.

Corollary 3.7. Let $(M, g(x))$ be a complete noncompact 3-dimensional manifold with bounded nonnegative sectional curvature $0 \leq R m \leq$ $K_{0}$, for some fixed constants $K_{0}$. Let $g(x, t)$ be a smooth complete solution to the Ricci flow on $M \times[0, T]$ with $g(x)$ as initial data. Then we have

$$
0 \leq R m(\cdot, t) \leq \frac{1}{\frac{1}{K_{0}}-4 t}
$$

for all $0 \leq t<\min \left\{T, \frac{1}{4 K_{0}}\right\}$.

Proof. First of all, since we are considering the curvature estimate, by pulling back the solution $g_{t}$ to the universal cover of the manifold, it is sufficient to assume the manifold is simply-connected. We claim that for such simply connected manifold, there is a constant $i_{0}>0$ (may depend on the initial curvature bound) such that the initial metric has injectivity radius bounded from below by $i_{0}>0$.

Actually, since the curvature is bounded, by [14], we may deform the initial metric by the Ricci flow in a short time interval $[0, \delta]$ such that the solution $\tilde{g}_{\tau}(\tau \in[0, \delta])$ has bounded and nonnegative curvature $0 \leq R m \leq 2 K_{0}$. Here the construction of $\tilde{g}$ is from [14], there should be no ambiguity with the given solution in our theorem. We have two possibilities. If there is $\tau>0$ such that the sectional curvature vanishes somewhere for some directions, then by the strong maximum principle of Hamilton, the manifold splits as $\mathbb{R} \times \Sigma$ or $\mathbb{R}^{3}$ metrically for all $\tau \in(0, \delta]$, where $\Sigma$ is surface with bounded and positive sectional curvature. Another case is for all $\tau \in(0, \delta]$, the sectional curvatures of the solution are positive everywhere. The following fact is standard: 
the injectivity radius of the manifold is bounded from below by $\frac{\pi}{\sqrt{C}}$, for simply connected closed even-dimensional manifold with $0<\sec \leq C$ or complete noncompact Riemannian manifold with $0<s e c \leq C$. So we know the injectivity radius is bounded from below by a uniform positive constant for any $0<\tau<\delta$. Then our assertion follows from the fact that the volume of the unit ball at $t=0$ is uniformally bounded (from below by a positive constant) by the Ricci flow equation.

Note that by Corollary 2.3(ii) the nonnegativity of sectional curvature is preserved for $t>0$. Since we have lower injectivity radius bound at time $t=0$ from above argument, then by applying Theorem 3.6, we know there is a constant $K>0$ depending only on $i_{0}$ and $K_{0}$ such that $|R m|(\cdot, t) \leq 2 K_{0}$ for $t \in\left[0, \min \left\{T, \frac{1}{K}\right\}\right]$. On the other hand, once the curvature is bounded, we can apply the maximum principle (on complete manifold with bounded curvature), yielding

$$
0 \leq R m(x, t) \leq \frac{1}{\frac{1}{K_{0}}-4 t} .
$$

Moreover we know the volume of the unit ball is also bounded from below as long as the curvature is bounded. So we may apply Theorem 3.6 and maximum principle estimate repeatedly. So (3.15) holds for all $0 \leq t<\min \left\{T, \frac{1}{4 K_{0}}\right\}$.

q.e.d.

Combining [3] and Corollary 3.7, we complete the proof of Theorem 1.1. The following theorem follows also as a corollary of $[\mathbf{3}]$ and Corollary 3.5.

Theorem 3.8. Let $(M, g(0))$ be a complete smooth 3 dimensional Riemannian manifold such that $|R m|(\cdot, 0) \leq K_{0}$ on $M$. Suppose we have two smooth complete solutions $g_{1}(t)$ and $g_{2}(t)$ to the Ricci flow $\left(g_{i j}\right)_{t}=-2 R_{i j}, 0 \leq t \leq T$, on $M$ with $g(0)$ as initial metric, and there is $C>0$ such that $g_{i}(\cdot, t) \geq \frac{1}{C} g(\cdot, 0)$ on $M \times[0, T](i=1,2)$, then we have $g_{1}(t)=g_{2}(t)$ for all $0 \leq t \leq T$.

In concluding this section, we discuss the two dimensional case. In this case, we can obtain purely local a priori estimates.

Proposition 3.9. Let $g(x, t), t \in[0, T]$ be a smooth solution to the Ricci flow with $g(x, 0)$ as initial metric on a two dimensional Riemannian manifold $M, x_{0} \in M$. We assume $B_{t}\left(x_{0}, r_{0}\right)$ is compactly contained in $M$ for any $t \in[0, T]$; and at $t=0,|R|(x, 0) \leq r_{0}^{-2}$ on $B_{0}\left(x_{0}, r_{0}\right)$ and $\operatorname{vol}_{0}\left(B_{0}\left(x_{0}, r_{0}\right)\right) \geq v_{0} r_{0}^{2}$ for some constants $r_{0}, v_{0}>0$. Then there is a constant $K$ depending only on $v_{0}$ such that

$$
|R|(x, t) \leq 2 r_{0}^{-2}
$$

for all $x \in B_{t}\left(x_{0}, \frac{r_{0}}{2}\right), 0 \leq t \leq \min \left\{T, \frac{1}{K} r_{0}^{2}\right\}$. 
Proof. The argument is similar to Theorem 3.6. After choosing the largest time $T_{0}$ such that curvature norm reaches $2 r_{0}^{-2}$ on the balls of radius $\frac{r_{0}}{2}$, by using Proposition 2.1 , we have curvature estimate $R(x, t) \geq$ $-C r_{0}^{-2}$ on balls of radius $\frac{3}{4} r_{0}$. Note that the dimension is two, scalar curvature is the only curvature we have, so this lower curvature bound enables us to apply the Bishop-Gromov volume comparison theorem. Therefore, in the rest, we can argue as in the proof of Theorem 3.6 to derive a lower bound for $T_{0}$.

q.e.d.

The following result is also clearly a corollary of Proposition 3.9.

Theorem 3.10. Let $(M, g(0))$ be a complete smooth 2 dimensional Riemannian manifold such that $|R| \leq K_{0}$, and $\operatorname{vol}_{0}\left(B_{0}(\cdot, 1)\right) \geq v_{0}$ for some fixed positive constants $K_{0}, v_{0}$. Suppose we have two smooth complete solutions $g_{1}(t)$ and $g_{2}(t)$ to the Ricci flow $\left(g_{i j}\right)_{t}=-2 R_{i j}$, $0 \leq t \leq T$, on $M$ with initial metric $g(0)$, then we have $g_{1}(t)=g_{2}(t)$, for $0 \leq t \leq \min \left\{T, \frac{1}{K_{0}}\right\}$.

\section{Concluding remarks}

It is interesting to know if the pseudolocality theorem of the Ricci flow holds in a general class of Riemannian manifolds, and the strong uniqueness theorem holds in general as the corollary. In particular, we may ask the question for Euclidean space $\mathbb{R}^{n}$ :

Question Does the strong uniqueness of the Ricci flow hold on the Euclidean space $\mathbb{R}^{n}$ for $n \geq 4$ ?

In the present paper, we have proved the case for $n=2$ and 3 .

We give remarks for the analogous results on mean curvature flow. We should mention that for codimension one hypersurfaces in Euclidean space, the same type estimate was firstly established by Ecker and Huisken $[6]$. There are much study for higher codimensional mean curvature flow, see M.T. Wang [16]. A pseudolocality estimate and general strong uniqueness theorem for mean curvature flow were obtained in [5]. The above question is an intrinsic version of the result in [5].

\section{References}

[1] H.D. Cao \& X.P. Zhu, A complete proof of Poincare and geometrization conjectures-application of Hamilton-Perelman theory of Ricci flow, Asian J. Math. 102 (2006), 165-492, MR 2233789, Zbl pre05071705.

[2] A. Chau, L.F. Tam \& C.J. Yu, Pseudolocality for the Ricci flow and applications, arXiv: math.DG/0701153.

[3] B.L. Chen \& X.P. Zhu, Uniqueness of the Ricci flow on complete noncompact manifolds, J. Differential Geom. 74 (2006), 119-154, MR 2260930, Zbl 1104.53032. 
[4] B.L. Chen \& X.P. Zhu, Ricci flow with Surgery on four-manifolds with positive isotropic curvature, J. Differential Geom. 74 (2006), 177-264. MR 2258799, Zbl 1103.53036.

[5] B.L. Chen \& L. Yin, Uniqueness and pseudolocality theorems of the mean curvature flow, Comm. Anal. Geom. 15 (2007), no.3, 435-490, MR 2379801, Zbl 1138.53055.

[6] K. Ecker \& G. Huisken, Interior estimates for hypersurfaces moving by mean curvature, Invent. Math. 105 (1991), 547-569, MR 1117150, Zbl 0707.53008

[7] R. S. Hamilton, Three manifolds with positive Ricci curvature, J. Differential Geom. 17 (1982), 255-306, MR 664497, Zbl 0504.53034.

[8] R. S. Hamilton, The formation of singularities in the Ricci flow, Surveys in Differential Geometry (Cambridge, MA, 1993), 2, 7-136, International Press, Combridge, MA,1995, MR 1375255, Zbl 0876.53030.

[9] R. S. Hamilton, Non-singular solutions to the Ricci flow on three manifolds, Comm. Anal. Geom. 1 (1999), 695-729, MR 1714939, Zbl 0939.53024.

[10] B. Kleiner \& J. Lott, Note on Perelman's paper, http://www.math.lsa.umich. edu/research/ricciflow/perelman.html.

[11] J.W. Morgan \& G. Tian, Ricci flow and the Poincare conjecture. arXiv: math.DG/0607607.

[12] G. Perelman, The entropy formula for the Ricci flow and its geometric applications, arXiv:math.DG/0211159 v1 November 11, 2002. Preprint.

[13] G. Perelman, Ricci flow with surgery on three manifolds arXiv: math.DG/ 0303109 v1 March 10, 2003. Preprint.

[14] W.X. Shi, Deforming the metric on complete Riemannian manifold, J. Differential Geom. 30 (1989), 223-301, MR 1001277, Zbl 0676.53044.

[15] M. Simon, Ricci flow of almost nonnegatively curved three manifolds, arXiv: math. DG/0612095 v1 Dec. 4, 2006. Preprint.

[16] M.T. Wang, The mean curvature flow smoothes Lipschitz submanifolds, Comm. Anal. Geom. 12 (2004), no. 3, 581-599, MR 2128604, Zbl 1059.53053.

Department of Mathematics Sun Yat-SEN University 510275

GuANG ZHOU

P.R. CHINA

E-mail address: mcscbl@mail.sysu.edu.cn 